\title{
Superlattice supertoughness of TiN/MN (M=V, Nb, Ta, Mo, and W): First-principles study
}

\author{
Hai Wang ${ }^{\mathrm{a}}$, Huazhi Zeng ${ }^{\mathrm{a}}$, Qingkun $\mathrm{Li}^{\mathrm{b}}$ and Jun Shen ${ }^{\mathrm{a} 1}$ \\ ${ }^{a}$ College of Materials Science and Engineering, Tongji University, Shanghai 201804, China \\ ${ }^{\mathrm{b}}$ Key Laboratory of Electrical Engineering, Heilongjiang University, Harbin 150080, China
}

In this study, a supertoughness effect is predicted through systematic first-principle calculations on structures and mechanical properties of transition metal nitride superlattices TiN/MN $(M=\mathrm{V}, \mathrm{Nb}, \mathrm{Ta}, \mathrm{Mo}$, and W). The TiN/WN superlattice has an in-plane Cauchy pressure as high as $296 \mathrm{GPa}$, which is $348 \%$ larger than that in $\mathrm{Ti}_{0.5} \mathrm{~W}_{0.5} \mathrm{~N}$ solid solutions. Supertoughness is also significantly affected by the coupling between macro- and micro-distortions in addition to previously reported valence electron concentration and atomic ordering effects. Present results indicate a route to develop superhard supertough superlattice coatings.

Keywords: Nitrides; Mechanical properties; Toughness; Density functional theory

\footnotetext{
${ }^{1}$ Corresponding author. Tel.: +86 2169581009

E-mail: junshen@tongji.edu.cn.
} 


\section{Introduction}

Transition metal nitride coatings (superlattice and nanocomposite) have drawn considerable attention in the last two decades owing to their excellent mechanical properties. ${ }^{[1]}{ }^{[2]}{ }^{[3]}{ }^{[4]}$ Coatings have been nano-engineered as single or multilayer multicomponent systems. ${ }^{[5]}$ Excellent mechanical properties are considered closely related to micro-structures. In 1987, single-crystal TiN/VN superlattice coating ${ }^{[6]}$ was first found to have superhardness of $55 \mathrm{GPa}$, which is significantly higher than that of constituent TiN and VN. This superlattice superhardness effect enhances hardness at small modulation periods. The effect is confirmed in a series of $\mathrm{TiN} / M \mathrm{~N}$ ( $M$, transition metal) superlattice coatings. For example, TiN/NbN superlattice coatings exhibit very high hardness, high toughness, and elasticity, which result in significant improvements in tribological performance. ${ }^{[7]} \mathrm{TiN} / \mathrm{TaN}$ and TiN/TaWN superlattice coatings have maximum hardness values of $40 \mathrm{GPa}$ at modulation period of $9 \mathrm{~nm}$ and $50 \mathrm{GPa}$ at modulation period of $5.6 \mathrm{~nm}$, respectively. ${ }^{[8]}$ Mechanical and tribological properties of multilayered TiN/CrN, TiN/MoN, TiN/NbN, and TiN/TaN coatings are found superior to those of TiN. ${ }^{[9]}$ Among them, TiN/CrN coating have the best overall performance, whereas TiN/TaN show highest abrasive wear resistance. ${ }^{[9]}$

The hardness of TiN/MoN multilayer nanostructured coatings increases with decreasing modulation period (monolayer thickness) as expected. ${ }^{[10]}$ Only one phase with FCC lattice ( $\mathrm{NaCl}$ structure) is formed in coating at a low substrate bias voltage of $40 \mathrm{~V}$ at monolayer thickness around $2 \mathrm{~nm}$. Increasing substrate bias voltage to $230 \mathrm{~V}$ causes the formation of two-phase constituted by cubic TiN and high-temperature $\gamma-\mathrm{Mo}_{2} \mathrm{~N}$ with phase ratio TiN/MoN equal to $90 / 10 .{ }^{[10]}$ The effects of nanolayer thickness on the structure and properties of multilayer TiN/MoN coatings were investigated. ${ }^{[1]}$ The hardness of TiN/MoN reaches a maximum of $29-31 \mathrm{GPa}$, which is significantly less than the hardness of MoN (36-40 GPa). ${ }^{[12]}$ Although no superhardness for TiN/MoN coatings have been reported, Nordin et al. showed that TiN/MoN coatings deposited on cemented carbide have a hardness $(29.7 \mathrm{GPa})$ comparable to that of TiN/NbN (31.9 GPa) and 
TiN/TaN (35.6 GPa). ${ }^{[9]}$ However, this is due to the fact that these coatings are polycrystalline: not deposited on single-crystal substrates and not coherently-grown with cube-on-cube relationship. For TiN/WN superlattice coatings, there is no experimental report. $\mathrm{Ti}_{0.5} \mathrm{~W}_{0.5} \mathrm{~N}$ solid solution thin films were grown on $\mathrm{MgO}(001)$ substrates, but no hardness values were reported. ${ }^{[13]}$ Thus, the superlattice superhard effect is highly expected to be experimentally confirmed in coherent-growth TiN/MoN and TiN/WN.

Although a large number of experimental investigations on preparation, deposition, hardness, and wear have been conducted, few studies have focused on the toughness of nitride coatings, which are important for industrial application and scientific research because the most important properties for tools for machining applications include not only hardness but also toughness (even chemical stability) ${ }^{[14]}$; especially for nanocomposite materials, high hardness need to be combined with high toughness (Ref.[15] and references therein). For TiN-based nitrides, Chen et al. ${ }^{[16]}$ revealed that Mo and $\mathrm{W}$ are the best alloying candidates to improve ductility in TiN-based nitrides. Considering these results, Zhao et al. ${ }^{[17]}$ mapped the hardness and ductility trends of binary and ternary metal nitrides to identify several potential ternary combinations for improved toughness. Recently, Sangiovanni et al. used first-principle calculations to examine the electronic mechanism for toughness enhancement in $\mathrm{Ti}_{\mathrm{x}} M_{1-\mathrm{x}} \mathrm{N}(M=\mathrm{Mo}$ and $\mathrm{W}) .{ }^{[18]}$ Then, they predicted that the valence electron concentration (VEC) enhances toughness in cubic $\mathrm{TiN}^{[18]} \mathrm{VN}^{[19]}$ and in $\mathrm{TiAlN}^{[20]}$ based alloys. Kindlund and coworkers, through their synthesis and testing of stoichiometric VMoN ${ }^{[21]}$ and VWN ${ }^{[22]}$ cubic single crystal, have shown the crystal to be both hard and tough. Toughness enhancements in hard single-crystal $\mathrm{V}_{0.5} \mathrm{Mo}_{0.5} \mathrm{~N}_{\mathrm{x}} / \mathrm{MgO}(001)$ thin films were also be confirmed by nanoindentation tests. ${ }^{[23]}$ An interesting issue is ensuring both hardness and toughness, which has great potential for industrial application. For example, Zhang et al. experimentally achieved superhard nanocomposites with sufficient toughness in nanocrystal-TiN/amorphous-(W,Ti) $\mathrm{C}_{0.83}$ superlattice films. ${ }^{[24]}$

This study investigates the structures, mechanical properties, and toughness of TiN/MN superlattices. A 
superlattice supertoughness effect is observed and its mechanism is discussed.

\section{Computational details and models}

Calculations were performed by applying density functional theory with generalized gradient approximation (GGA) in the scheme of Perdew-Burke-Ernzerhof. ${ }^{[25]}$ The ion-electron interaction was modeled by projector augmented wave $(\mathrm{PAW})^{[26,27]}$ in VASP. ${ }^{[28,29]}$ A plane-wave cutoff energy of $500 \mathrm{eV}$ was employed throughout the calculation. For the sampling of the Brillouin zone, structural optimization and mechanical properties of $\mathrm{TiN}$ and $M \mathrm{~N}$ were used in the $8 \times 8 \times 8$ and $30 \times 30 \times 30$ Monkhorst-Pack ${ }^{[30]} k$-point grids, respectively. For the following superlattice models, the same density $k$-point meshes are used. Whenever possible, we crosschecked the results by using $\mathrm{DMol}^{3}$, which uses density functional theory with a numerical radial function ${ }^{[31]}$ basis set to calculate the electronic properties of molecules, clusters, surfaces and crystalline solid materials ${ }^{[32]}$ from first principles. The consistency of our results for two sets of calculations is satisfactory.

The structural model of TiN/MN superlattices was constructed as follows. We first built a $2 \times 2 \times 2$ cubic-TiN supercell with 64 atoms. Then, we substituted some Ti atoms with $M$-atoms according to the following two models. Finally, we redefined its symmetry and applied the found space group. This is the function of "Find Symmetry" implemented in Materials studios. We use two structural models to examine the toughness of $\mathrm{TiN} / M \mathrm{~N}$ superlattices. In the first model, one TiN layer is replaced by one $M \mathrm{~N}$ layer along the [001] direction, which is consistent with the model of C\#1 in Ref.[18] and Ref.[33]. This model has a space group of P4/MMM (No. 123) with the cell of $(a / \sqrt{2}, a / \sqrt{2}, 2 a)$ TiN, containing one Ti $(0,0,0)$, one $M$ $(0.5,0.5,0.5)$, and two $\mathrm{N}(0,0,0.5$ and $0.5,0.5,0)$. This model contains the maximum number of intermetallic bonds. The second model C\#3 has a tetragonal space group of P42/MMC (No. 131), which has a cell of $(\sqrt{2} \mathrm{a}, \sqrt{2} \mathrm{a}, 2 a)$ TiN with one Ti $(0.25,0.5,0)$, one $M(0.75,0,0)$, and two $\mathrm{N}(0.25,0,0.5$ and $0.75,0.5$, 0.5). The structure of two models are depicted in Fig. 1. Fully structural optimization, including lattice 
constant and atomic coordination, were performed. Then, VASP code was used to calculate elastic tensor by performing six finite distortions of the lattice and deriving the elastic constants from the strain-stress relationship. ${ }^{[34]}$ Consulting Ref. [35], Cauchy pressure $\left(\mathrm{C}_{12}-\mathrm{C}_{44}\right)$ for tetragonal symmetry is calculated by $\mathrm{C}_{12}-\mathrm{C}_{44}=\hat{c}_{12}-\frac{1}{2}\left(\hat{c}_{44}+\hat{c}_{55}\right)$.

In this paper, we used Cauchy pressures, an indication of ductility, to examine the toughness. For a crystal with cubic symmetry, this is an easy defined: Cauchy pressure, $\mathrm{CP}=\mathrm{C}_{12}-\mathrm{C}_{44}$. However, this is not well defined for crystals with other symmetries. The general way to calculate Cauchy pressure for a non-cubic crystal is to project the elastic tensor into the closest cubic components ${ }^{[36]}$, and has been applied by Edström et al. to TMN with general crystal symmetry ${ }^{[37]}$. Edstrom and coworkers have provided a definition of averaged $\mathrm{CP}\left(\mathrm{CP}_{\mathrm{ave}}\right)$, which accounts for all crystallographic directions. It is $\mathrm{CP}_{\mathrm{ave}}=\mathrm{C}_{12}^{\prime}-\mathrm{C}_{44}^{\prime}$, here, $\mathrm{C}_{12}^{\prime}=\left(\mathrm{C}_{12}+\mathrm{C}_{13}+\mathrm{C}_{23}\right) / 3$ and $\mathrm{C}_{44}^{\prime}=\left(\mathrm{C}_{44}+\mathrm{C}_{55}+\mathrm{C}_{66}\right) / 3$. Because tetragonal symmetry have two equal "shear elastic constants $\left(\mathrm{C}_{44}=\mathrm{C}_{55}\right.$ and $\left.\mathrm{C}_{66}\right)$ " and two equal "tensile-compliant elastic constants $\left(\mathrm{C}_{12}=\mathrm{C}_{23}\right.$ and $\left.\mathrm{C}_{13}\right)$ ". Hence, $\mathrm{CPave}=\left(2 \mathrm{C}_{12}+\mathrm{C}_{13}\right) / 3-\left(2 \mathrm{C}_{44}+\mathrm{C}_{66}\right) / 3$. In additions, one can also define in-plane Cauchy pressure $\mathrm{CP}_{/ /}$and an out-plane Cauchy pressure $\mathrm{CP}_{\perp}$ as follows: $\mathrm{CP}_{/ /}=\mathrm{C}_{12}-\mathrm{C}_{44}$ and $\mathrm{CP}_{\perp}=\mathrm{C}_{13}-\mathrm{C}_{66}$.

\section{Results and discussion}

First, we performed the structural optimization of mononitrides $\mathrm{TiN}$ and $M \mathrm{~N}(M=\mathrm{V}, \mathrm{Nb}, \mathrm{Ta}, \mathrm{Mo}$, and W). Table I lists the results. The lattice constant of TiN, our theoretical value of $4.252 \AA$, agrees well with the previous theoretical value of $4.254 \AA^{[18]}$ and experimental one $(4.240 \AA)^{[38]}$. The lattice constants of VN, NbN, and TaN agree well with other theoretical reports (see Table I) and experimental ones (VN (4.15 $)$, ${ }^{[39]}$ $\mathrm{NbN}(4.442 \AA),{ }^{[40]}$ and TaN $\left.(4.331 \AA){ }^{[41]}\right)$. Note that both stoichiometric cubic WN and MoN are unstable at ambient conditions and there are also no experimental report on their lattice constants. Our theoretical equilibrium lattice constant of $\mathrm{WN}$ is consistent with previous theoretical prediction. ${ }^{[42]}$

The mechanical properties (including moduli and elastic constants) were calculated by using fully 
optimized structures. Bulk modulus $\mathrm{B}$, shear modulus $\mathrm{G}$ of $\mathrm{TiN}$, and $M \mathrm{~N}(M=\mathrm{V}, \mathrm{Nb}$, and Ta) agree well with previous reports. $\mathrm{C}_{\mathrm{ij}}$ of $\mathrm{VN}$ coincided with results of $\mathrm{DMol}^{3}$, which uses density functional theory with a numerical radial function basis set. ${ }^{[31]}$ For $\mathrm{NbN}$ and $\mathrm{TaN}, \mathrm{C}_{\mathrm{ij}}$ are in DFT error. The difference can be attributed to the difference of the DFT package.

Cauchy pressure $\left(\mathrm{C}_{12}-\mathrm{C}_{44}\right)$ has been extensively used to characterize the toughness of materials. ${ }^{\text {[18] [48] }}$ ${ }^{[49]}$ Our theoretical value for TiN is $-39 \mathrm{GPa}$, which is slightly less than the previously reported value of -44 GPa (Table I), indicating that TiN is brittle. ${ }^{[18]}{ }^{[48]}$ By contrast, all other mononitrides $M \mathrm{~N}$ with VB metals $(M=\mathrm{V}, \mathrm{Nb}$, and $\mathrm{Ta})$ are ductile because of their positive values of Cauchy pressure. For VN, Cauchy pressure

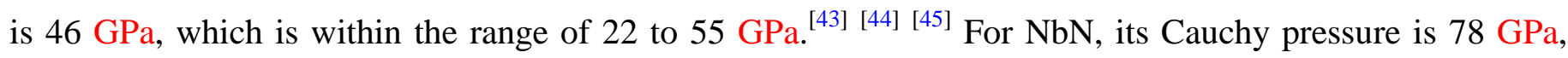
which is higher than all the previous reports of 49 to $58 \mathrm{GPa}^{[43]}{ }^{[44]}$ This value is dependent significantly on $k$-point meshes. We used $30 \times 30 \times 30$, which is clearly higher than $12 \times 12 \times 12$ used in previous reports. For

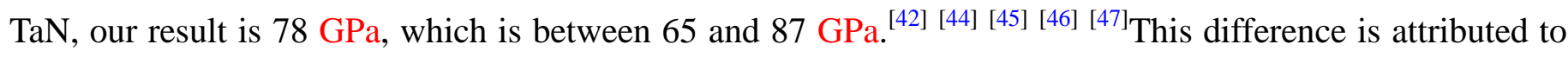
the different calculation parameters, such as energy cutoff and $k$-point meshes in previous investigations. In this study, we do not discuss the $\mathrm{C}_{\mathrm{ij}}$ and Cauchy pressure of $M \mathrm{~N}$ with VIB metals ( $M=\mathrm{Mo}$ and $\left.\mathrm{W}\right)$ because of their instability.

Now, we turn to TiN/MN superlattices. Table II shows lattice parameters, bulk modulus, shear modulus, elastic constant, and Cauchy pressure (both in-plane and out-plane as well as average Cauchy pressure) of TiN/MN superlattices in C\#1 model with space group of P4/MMM (No. 131). Our theoretical lattice constant $c$ of superlattices agrees well with that of solid solutions, ${ }^{[19]}$ and that of TiN/WN is close to corresponding experimental report on $\mathrm{Ti}_{1-\mathrm{x}} \mathrm{W}_{\mathrm{x}} \mathrm{N} \cdot{ }^{[13]}$ These superlattices have lattice constant values that are close to the arithmetic means of TiN and $M \mathrm{~N}$.

The Cauchy pressure and G/B ratio of TiN/MN superlattices are also presented in Fig. 2. The corresponding value of $\mathrm{Ti}_{0.5} M_{0.5} \mathrm{~N}$ solid solutions are given for comparison. We check first in-plane Cauchy 
pressure. For C\#1 superlattices, the in-plane Cauchy pressures of TiN/MN superlattices are $179 \%$ (TiN/VN)-482\%(TiN/NbN) larger than that of the corresponding solid solution. When $M$ changed from $\mathrm{V}$ to $\mathrm{W}$, the in-plane Cauchy pressure of $\mathrm{C \# 1}$ superlattices have a similar toughness trend as that in solid solutions, which has been attributed to the increase of VEC. ${ }^{[19]}$ For C\#3 superlattices, they also have the more larger in-plane Cauchy pressure and have the same trend as $\mathrm{C \# 1}$ ones. One can conclude that the toughness mechanism of our superlattices is partially similar to solid solutions. Note that the extremely high Cauchy pressure of up to $482 \%$ is reported for the first time in the present study.

Fig.2a clearly shows that in-plane Cauchy pressure $\mathrm{CP}_{/ /}$values are highly positive, while out-plane Cauchy pressure $\mathrm{CP}_{\perp}$ are highly negative, especially for these superlattices with $\mathrm{VB} M$ ions (V, Nb and Ta). Hence, there is giant anisotropy in toughness in these superlattices. This means that the supertoughness effect in the superlattices here we studied are ONLY for in-plane and NOT out-plane! The negative values of out-plane Cauchy pressure indicate TiN/MN superlattices are brittle, especially for the superlattices with VB metals. For the same direction of out-plane, all superlattices have high shear-over-bulk moduli ratio with negative Cauchy pressure. This indicates that ductile and compliant to deformation in one direction (in-plane) while stiff and strong/hard in the other direction (out-plane). Niu and coworkers ${ }^{[50]}$ have find similar case in $\mathrm{Al} X_{2}$ intermetallic compounds and suggested extra-electron induced covalent strengthening.

In addition, compared to the corresponding solid solutions, the average Cauchy pressures values reduce slightly for TiN/VN and TiN/TaN superlattices, and increase significantly in TiN/NbN, TiN/MoN and TiN/WN ones. This suggests that the later three superlattices still be potential supertoughness coatings.

Fig. $2 \mathrm{~b}$ shows that the toughness (in this case, $\mathrm{G} / \mathrm{B}$ ) of superlattices $\mathrm{C} \# 1$ has a similar trend as that of solid solutions (except TiN/TaN), and its value outnumberes that of solid solutions by not more than $25 \%$. In this case, superlattice $\mathrm{C \# 1}$ shows less toughness than the corresponding solid solution. While G/B criteria are related to bulk effect, Cauchy pressure is related to the detailed transform under stress. Thus, the 
different criteria might result in different toughness trends.

For C\#3 superlattices, Fig. 2a shows that the in-plane Cauchy pressure of TiN/VN improved slightly, whereas that of TiN/WN dropped. The criterion G/B ratio indicates that the toughness of $\mathrm{C \# 3} \mathrm{TiN} / M \mathrm{~N}$ superlattices is improved compared to $\mathrm{C \# 1}$ superlattices, especially for TiN/MoN and TiN/WN (Fig. 2b). The trend of toughness becomes more complex than that in the C\#1 superlattice and solid solution.

According to $\mathrm{Pugh}^{[51]}$ and Pettifor ${ }^{[52]}$ criteria, we mapped the ductility trend of $\mathrm{C \# 1}$ TiN/MN superlattices and $\mathrm{Ti}_{0.5} M_{0.5} \mathrm{~N}$ solid solutions. Fig. 3a,c presents the results. The in-plane Cauchy pressures indicate that TiN/MN superlattices within the ductile region of the map (the yellow area) are TiN/MoN and TiN/WN. Besides, $\mathrm{Ti}_{0.5} \mathrm{Ta}_{0.5} \mathrm{~N}$ is clearly observed within the ductile region, but TiN/TaN is not. This observation means that the superlattice supertoughness effect is present only in TiN/MoN and TiN/WN. Compared to the superlattices with VB metals, TiN/MN superlattices containing VIB metals have the more large toughness in terms of the criteria of Cauchy pressure proposed by Pettifor and the more low Pugh's modulus ratio of G/B. This can be attributed to the increased valence electron concentration. ${ }^{[19]}$ To observe the changes of G/B ratio and Cauchy pressure directly, we calculated the difference value between TiN/MN superlattices and $\mathrm{Ti}_{0.5} M_{0.5} \mathrm{~N}$ solid solutions. Fig. $3 \mathrm{~b}$ indicates the results. Obviously, except $\mathrm{V}$, the values of $\delta_{\mathrm{C} 12-\mathrm{C} 44}$ above 100 enhanced ductility significantly. The large $\delta_{(\mathrm{G} / \mathrm{B})}$ of Ta results in the disappearance of TiN/TaN in the ductile region. Fig. 3c depicts the out-plane Cauchy pressure and G/B. It is evident that superlattices with VB metals move to brittle region, while TiN/MoN and TiN/WN superlattice move out ductile region.

The results shown in Figs. 2 and 3 are unusual because we used the same atomic ordering employed by Sangiovanni et al. ${ }^{[18]}$ The difference is that we reconstructed the structural symmetry in tetragonal phase, which results in the fact that the macro-distortion (in this case, we defined it as the ratio $(c / a)$ of lattice constant $c$ and $a$.) and/or micro-distortions are possible in our calculations. Until today, the phenomenon of 
supertoughness and its mechanism in superlattice coating systems are unknown, but will be explained in the following discussion.

Properties of materials are determined by their structures. Thus, we first checked the structures of TiN/MN superlattices with two configurations (listed in Tables III and IV, respectively). For both configurations with different atomic ordering, lattice constants $a$ and $c$ first increase and then decrease for VB (from V, $\mathrm{Nb}$ to Ta) superlattices, while $a(c)$ increase (decrease) for superlattices with VIB (from Mo to W) metals. In the C\#1 model, all bond-length including Ti- $M$, Ti-N/M-N(xy), and Ti-N/M-N(z) have the same trend as lattice constant $a$ for VB superlattices. For VIB superlattices Ti- $M$ and Ti-N/M-N(xy), bond-lengths have a trend of lattice constant $a$, and Ti-N/M-N(z) has a trend of lattice constant $c$. In the C\#3 superlattices, Ti-Ti and $M-\mathrm{N}$ bond-lengths have the same trend as the $\mathrm{C} \# 1$ ones, and the $M-M$ bond-length decreases. However, the bond-length of Ti-N increases. The bond-length of Ti-M in C\#1 model fell within the range of $[2.96,3.08]$ (see Table 3), while the Ti-Ti and $M-M$ are within the range of $[2.96,3.18]$ for C\#3 model (see Table 4). The $M-\mathrm{N}(\mathrm{xy})$ bond-lengths decreases for VIB metals in C\#1 and remains unchanged in the C\#3 model. The Me-N(z) bond-lengths increase when $M$ was considered as VB metal for C\#1 and C\#3 superlattices, while remaining unchanged for VIB metals. In one word, the micro-structure of superlattices is very different to the corresponding solid solutions, which have high symmetry (such as lattice constant $a=b=c, M-\mathrm{N}$ bonds have the same bond-length as Ti-N).

In this case, we defined lattice constant change (the ratio of $c / a$ ) as macro-distortion, while local atomic change corresponded to micro-distortion. Interestingly, the C\#1 model has only a macro-strain distortion (i.e., cla changed, but atomic coordination did not). Meanwhile, the C\#3 model has both macro- and micro-distortions (both $c / a$ and atomic coordination changed). Thus, two model superlattices are ideal prototypes for investigating the influence of structural distortions. The macro-distortion decreases for all superlattices with VB and VIB transition metals (Table III and IV). In the C\#3 model, structural optimization 
results in the following atomic coordination changes: Ti atoms from $(0.25,0.5,0)$ to $(0.24,0.5,0), M$ atoms from $(0.75,0,0)$ to $(0.72,0,0)$, and two $\mathrm{N}$ atoms from $(0.25,0,0.5)$ to $(0.24,0,0.5)$ and $(0.75,0.5,0.5)$ to $(0.73,0.5,0.5)$, respectively. Fig. 4 depicts the structures of TiN/MoN superlattice before and after optimization. Local atomic micro-distortions are evident.

In terms of total energy, the C\#3 model has the lower total energy (up to $0.4 \mathrm{eV} /$ cell) than $\mathrm{C \# 1}$ (except TiN/WN), which is consistent with previous research. ${ }^{[18]}$ Previous density functional theory calculations have demonstrated that atomic ordering has negligible influence on lattice constants of $\mathrm{Ti}_{0.5} \mathrm{Mo}_{0.5} \mathrm{~N}$ and $\mathrm{Ti}_{0.5} \mathrm{~W}_{0.5} \mathrm{~N}$ (Table I of Ref. [37]). However, for TiN/WN superlattice, its C\#1 model rather than C\#3 has the lower total energy, which indicates that atomic ordering has a strong influence in this case. Thus, coupling is observed between macro- and micro-distortion. In fact, Wang et al. have reported similar phenomena in simple cubic perovskites $\mathrm{Bi} \mathrm{OO}_{3}{ }^{[53]}$, and $\mathrm{B}$-site complex perovskites $\mathrm{Bi}_{2} \mathrm{ZnTiO}_{6}{ }^{[54]}$ had superferroelectricity and superbirefringent crystals $A \mathrm{FBiS}_{2}{ }^{[55]}$

Table V indicates that macro-distortion significantly improves the in-plane toughness of the superlattice, whereas the effects of micro-distortion on the in-plane toughness is different for TiN/MN (TiN/VN rising, others falling). The coupling of macro- and micro-distortions significantly affects their in-plane toughness (see Fig. 2a). Specifically, C\#1 TiN/WN superlattice has in-plane Cauchy pressure as high as $296 \mathrm{GPa}$, which is $348 \%$ larger than that in the $\mathrm{Ti}_{0.5} \mathrm{~W}_{0.5} \mathrm{~N}$ solid solution ${ }^{[18]}$ and is the largest value ${ }^{[37]}$ among obtainable results for nitride coatings.

To show the influence of macro- and micro-distortions on toughness of superlattice, we performed a series of structural optimization and mechanical calculations on mono-nitrides $\mathrm{TiN}$ and $M \mathrm{~N}(M=\mathrm{V}, \mathrm{Nb}, \mathrm{Ta}$, Mo, and W) constrained with in-plane lattice constant $a$ of the corresponding superlattice. While constraint-free TiN/MN C\#1 superlattices have only macro-distortion as mentioned previously, constrained superlattices can have both macro- and micro-distortions. Fig. 5 depicts the lattice constant $c$ of TiN, $M \mathrm{~N}$, 
and TiN/MN superlattices at corresponding superlattice constant $a$. First, the appearance of lattice constant $c$ of the superlattice systems between that of $\mathrm{TiN}$ and $M \mathrm{~N}$ is easy to understand. For TiN, the lattice constant $c$ decreases as in-plane lattice constant $a$ increases. This finding indicates the well-known phenomenon determined by Poisson ratio. Note that the constraint of substrate-made thin films have a cube-on-cube growth, i.e., the out-plane lattice constant of the thin film with constraint should be different from that of bulk materials. The superlattices with VIB metal are located between TiN/VN and TiN/Nb. This finding is consistent with the lattice constant trend of $\mathrm{VN}<\mathrm{MoN}<\mathrm{WN}<\mathrm{NbN}<\mathrm{TaN}$ as shown in Table I. For all superlattices, their $c$ increases linearly for contained VB metal and decreases for VIB metal. Note that TiN/VN is different from other SLs because the $c$ of VN is less than that of TiN, while the other MN is larger than TiN. Compared to lattice constants of constraint-free TiN, $M \mathrm{~N}$, and TiN/MN C\#1 superlattices, micro-distortion introduced by the superlattice constant results in changes in constrained TiN/NbN and TiN/WN. The influence of the macro- and micro-distortion coupling effects on the structures of the superlattice is visible (Fig. 5a and Fig. 5b).

Figure 6 shows the Cauchy pressure $\mathrm{CP}\left(=\mathrm{C}_{12}-\mathrm{C}_{44}\right)$ of $\mathrm{TiN}, M \mathrm{~N}$, and in-plane Cauchy pressure $\mathrm{CP}_{\|}(=$ $\left.\mathrm{C}_{12}-\mathrm{C}_{44}\right) \mathrm{TiN} / M \mathrm{~N}$ superlattices at corresponding superlattice constant $a$. The Cauchy pressure of TiN has minimal change as superlattice constant $a$ increases. This observation does not hold true for other mononitrides. The Cauchy pressure of $M \mathrm{~N}$ with $\mathrm{VB}$ metals ( $\mathrm{V}, \mathrm{Nb}$, and $\mathrm{Ta}$ ) is within the range of 50-80 GPa, while that of the $M \mathrm{~N}$ with VIB metals (Mo and W) is as large as 295-363 GPa. Interestingly, superlattices with VB metals ( $\mathrm{V}, \mathrm{Nb}$, and $\mathrm{Ta}$ ) have higher in-plane Cauchy pressure than that of both constituents TiN and $M \mathrm{~N}$ (right panel of Fig. 6). Thus, their superlattices have in-plane supertoughness. Although the in-plane Cauchy pressure of TiN/MN superlattices with VIB metals (TiN/MoN and TiN/WN) is less than that of $M \mathrm{~N}$, their absolute values (170-228 GPa) are larger than the largest value (85 GPa, Fig. 2) of the $\mathrm{Ti}_{0.5} M_{0.5} \mathrm{~N}$ solid solution. In this context, the VIB metal superlattice similarly exhibits in-plane supertoughness. Compared 
with the linear relation (Fig. 6b) of constrained TiN/MN superlattices with VB metals, TiV/NbN has an extra increase as shown in Fig. 2a. The coupling of macro- and micro-distortion has positive (negative) influence on the in-plane toughness of superlattices with VB (VIB) metals. TiN/MN superlattices in the present study exhibit in-plane superlattice supertoughness, as their superlattice superhard effect was reported first in 1987.

In addition, as Sangiovanni D. G. et al. ${ }^{[56]}$ suggested, the empirical criteria of $\operatorname{Pugh}^{[51]}$ and Pettifor ${ }^{[52]}$ are based solely on the values of elastic constants, while plastic deformation in solids primarily occurs beyond the elastic-response regime. Thus, it gives indications of the mechanical behavior only within the elastic-response regime. To really check whether the material is ductile/plastic one should calculate stress-strain curves up to and beyond the yield point. Plasticity/ductility can be seen by a bowing of stress-strain curves. For TiN/WN superlattices, these results have been published in Ref.[56]. The stress-strain curves for $\{110\}<110>$ and $\{110\}<110>$ slip systems of TiN/WN superlattice demonstrates that superlattice deform plastically beyond the yield point. In this context, the toughness of TiN/MN superlattices are highly expected by furthermore study in this direction.

The mechanism of supertoughness is important for developing new superhard and supertough superlattice coatings. Previously (in 2011), the supertoughness of TiN and VN alloy with transition metals $(M=\mathrm{Nb}, \mathrm{Ta}, \mathrm{Mo}$, or $\mathrm{W})$ is attributed to the increased valence electron concentration (VEC). ${ }^{[19]}$ Thereafter (in 2014), atomic ordering is demonstrated to have a significant effect on the toughness of solid solutions $\mathrm{V}_{0.5} \mathrm{~W}_{0.5} \mathrm{~N}, \mathrm{~V}_{0.5} \mathrm{Mo}_{0.5} \mathrm{~N}, \mathrm{Ti}_{0.5} \mathrm{~W}_{0.5} \mathrm{~N}$, and $\mathrm{Ti}_{0.5} \mathrm{Mo}_{0.5} \mathrm{~N}{ }^{[37]}$ In this case, our results demonstrate that toughness is closely related to the coupling of the macro-strain and micro-distortion. Macro-strain can be easily introduced by substrates; thus, our findings reveal that structural design is a new way to obtain supertough and superhard superlattice coatings. In addition, it is expected to account for the surface effect in the further studies of mechanical properties on coatings, because an earlier report ${ }^{[57]}$ shows that surface condition and environment can significantly affect strength and ductility. 


\section{Conclusions}

In summary, we performed first-principle calculations on the structural and elastic-properties of TiN/MN superlattices. Our theoretical structural parameters of mononitrides TiN and $M \mathrm{~N}$ agree well with previous reports. Present results indicate a supertoughening effect in $\mathrm{TiN} / M \mathrm{~N}$ superlattices due to the combination of enhanced in-plane ductility with preserved out-of-plane mechanical strength. The mechanism is shown to be closely related to the coupling between macro- and micro-distortions as well as previously reported valence electron concentration (VEC) and atomic ordering effects. Our results suggest a route to developing superhard and supertough superlattice coatings through proper structural design in chemical composition (VEC), macro-structure (substrate constraint), and micro-structure (atomic ordering).

\section{Acknowledgements}

This work was supported by the National Science Foundation of China under grant No.U1302275. 


\section{References}

[1] J. Musil, Hard and superhard nanocomposite coatings, Surface \& Coatings Technology, 125 (2000) 322-330.

[2] S. Veprek, A.S. Argon, Towards the understanding of mechanical properties of super- and ultrahard nanocomposites, Journal of Vacuum Science \& Technology B, 20 (2002) 650-664.

[3] F. Vaz, L. Rebouta, P. Goudeau, et al., Characterisation of Ti1-xSixNy nanocomposite films, Surface \& Coatings Technology, 133 (2000) 307-313.

[4] S. Veprek, S. Reiprich, A concept for the design of novel superhard coatings, Thin Solid Films, 268 (1995) 64-71.

[5] S. PalDey, S.C. Deevi, Single layer and multilayer wear resistant coatings of (Ti,Al)N: a review, Mater. Sci. Eng. A, 342 (2003) 58-79.

[6] U. Helmersson, S. Todorova, S.A. Barnett, et al., Growth of single-crystal TiN/VN strained-layer superlattices with extremely high mechanical hardness, Journal of Applied Physics, 62 (1987) 481-484.

[7] X.T. Zeng, TiN/NbN superlattice hard coatings deposited by unbalanced magnetron sputtering, Surface and Coatings Technology, 113 (1999) 75-79.

[8] X. Junhua, L. Geyang, G. Mingyuan, The microstructure and mechanical properties of TaN/TiN and TaWN/TiN superlattice films, Thin Solid Films, 370 (2000) 45-49.

[9] M. Nordin, M. Larsson, S. Hogmark, Mechanical and tribological properties of multilayered PVD TiN/CrN, TiN/MoN, TiN/NbN and TiN/TaN coatings on cemented carbide, Surface \& Coatings Technology, 106 (1998) 234-241.

[10] A.D. Pogrebnjak, G. Abadias, O.V. Bondar, et al., Structure and Properties of Multi layer Nanostructured Coatings TiN/MoN Depending on Deposition Conditions, Acta Physica Polonica A, 125 (2014) 1280-1283.

[11] A.D. Pogrebnjak, V.M. Beresnev, O.V. Bondar, et al., The effect of nanolayer thickness on the structure and properties of multilayer TiN/MoN coatings, Technical Physics Letters, 40 (2014) 215-218.

[12] V. Beresnev, O. Bondar, B. Postolnyi, et al., Comparison of tribological characteristics of nanostructured TiN, MoN, and TiN/MoN Arc-PVD coatings, Journal of Friction and Wear, 35 (2014) 374-382.

[13] F. Tian, J. D'Arcy-Gall, T.-Y. Lee, et al., Epitaxial Ti1-xWxN alloys grown on $\mathrm{MgO}(001)$ by ultrahigh vacuum reactive magnetron sputtering: Electronic properties and long-range cation ordering, J. Vac. Sci. Technol. A, 21 (2003) 140-146.

[14] M.G. Faga, G. Gautier, R. Calzavarini, et al., AlSiTiN nanocomposite coatings developed via Arc Cathodic PVD: Evaluation of wear resistance via tribological analysis and high speed machining operations, Wear, 263 (2007) 1306-1314.

[15] S. Vepřek, The search for novel, superhard materials, Journal of Vacuum Science \& Technology A, 17 (1999) 2401-2420.

[16] K. Chen, L. Zhao, J. Rodgers, et al., Alloying effects on elastic properties of TiN-based nitrides, Journal of Physics D: Applied Physics, 36 (2003) 2725.

[17] L. Zhao, K. Chen, Q. Yang, et al., Materials informatics for the design of novel coatings, Surface and Coatings Technology, 200 (2005) 1595-1599.

[18] D.G. Sangiovanni, V. Chirita, L. Hultman, Electronic mechanism for toughness enhancement in TixM1-xN (M=Mo and W), Phys. Rev. B, 81 (2010) 104107.

[19] D.G. Sangiovanni, L. Hultman, V. Chirita, Supertoughening in B1 transition metal nitride alloys by increased valence electron concentration, Acta Mater., 59 (2011) 2121.

[20] D. Sangiovanni, V. Chirita, L. Hultman, Toughness enhancement in TiAlN-based quarternary alloys, Thin Solid Films, 520 (2012) 4080-4088. 
[21] H. Kindlund, D. Sangiovanni, L. Martínez-de-Olcoz, et al., Toughness enhancement in hard ceramic thin films by alloy design, APL Materials, 1 (2013) 042104.

[22] H. Kindlund, D. Sangiovanni, J. Lu, et al., Effect of WN content on toughness enhancement in V1- xWxN/MgO (001) thin films, Journal of Vacuum Science \& Technology A, 32 (2014) 030603.

[23] H. Kindlund, D.G. Sangiovanni, J. Lu, et al., Vacancy-induced toughening in hard single-crystal V0.5Mo0.5Nx/MgO(001) thin films, Acta Mater., 77 (2014) 394-400.

[24] S.H. Zhang, E. Byon, M.X. Li, et al., Realization of superhard nanocomposites with sufficient toughness: Superlattice nanocrystal-TiN/amorphous-(W,Ti)C-0.83 films, Thin Solid Films, 519 (2011) 1901-1906.

[25] J.P. Perdew, K. Burke, M. Ernzerhof, Generalized Gradient Approximation Made Simple, Phys. Rev. Lett., 77 (1996) 3865-3868.

[26] P.E. Blöchl, Projector augmented-wave method, Phys. Rev. B, 50 (1994) 17953-17979.

[27] G. Kresse, D. Joubert, From ultrasoft pseudopotentials to the projector augmented-wave method, Phys. Rev. B, 59 (1999) 1758-1775.

[28] G. Kresse, J. Furthmüller, Efficiency of ab-initio total energy calculations for metals and semiconductors using a plane-wave basis set, Comp. Mater. Sci., 6 (1996) 15-50.

[29] G. Kresse, J. Furthmüller, Efficient iterative schemes for ab initio total-energy calculations using a plane-wave basis set, Phys. Rev. B, 54 (1996) 11169-11186.

[30] H.J. Monkhorst, J.D. Pack, Special points for Brillouin-zone integrations, Phys. Rev. B, 13 (1976) 5188-5192.

[31] B. Delley, An all-electron numerical method for solving the local density functional for polyatomic molecules, The Journal of Chemical Physics, 92 (1990) 508-517.

[32] B. Delley, From molecules to solids with the DMol3 approach, The Journal of Chemical Physics, 113 (2000) 7756-7764.

[33] P. Mayrhofer, D. Music, J. Schneider, Influence of the Al distribution on the structure, elastic properties, and phase stability of supersaturated Ti 1- x Al x N, J. Appl. Phys, 100 (2006) 094906.

[34] Y. Le Page, P. Saxe, Symmetry-general least-squares extraction of elastic data for strained materials from Itextit\{ab initio \} calculations of stress, Phys. Rev. B, 65 (2002) 104104.

[35] M. Moakher, A.N. Norris, The closest elastic tensor of arbitrary symmetry to an elasticity tensor of lower symmetry, J. Elasticity., 85 (2006) 215-263.

[36] F. Tasnádi, M. Odén, I.A. Abrikosov, Ab initio elastic tensor of cubic Ti $0.5 \mathrm{Al} 0.5 \mathrm{~N}$ alloys: dependence of elastic constants on size and shape of the supercell model and their convergence, Phys. Rev. B, 85 (2012) 144112.

[37] D. Edström, D.G. Sangiovanni, L. Hultman, et al., Effects of atomic ordering on the elastic properties of TiN- and VN-based ternary alloys, Thin Solid Films, 571 (2014) 145-153.

[38] N. Pessall, R.E. Gold, H.A. Johansen, A study of superconductivity in interstitial compounds, J. Phys. Chem. Solids, 29 (1968) 19-38.

[39] B.R. Zhao, L. Chen, H.L. Luo, et al., Superconducting and normal-state properties of vanadium nitride, Phys. Rev. B, 29 (1984) 6198-6202.

[40] R.E. Treece, M.S. Osofsky, E.F. Skelton, et al., New phase of superconducting NbN stabilized by heteroepitaxial film growth, Phys. Rev. B, 51 (1995) 9356-9359.

[41] J. Urban, Thermal diffuse X-ray scattering for small samples and small coherent scattering domains, Acta Crystallogr. A, 31 (1975) 95-100. 
[42] H. Rached, D. Rached, S. Benalia, et al., First-principles study of structural stabilities, elastic and electronic properties of transition metal monocarbides (TMCs) and mononitrides (TMNs), Mater. Chem. Phys., 143 (2013) 93-108.

[43] B.D. Fulcher, X.Y. Cui, B. Delley, et al., Hardness analysis of cubic metal mononitrides from first principles, Phys. Rev. B, 85 (2012) 184106.

[44] V. Petrman, J. Houska, Trends in formation energies and elastic moduli of ternary and quaternary transition metal nitrides, J. Mater. Sci., 48 (2013) 7642-7651.

[45] D. Holec, M. Friák, J. Neugebauer, et al., Trends in the elastic response of binary early transition metal nitrides, Phys. Rev. B, 85 (2012) 064101.

[46] S.K.R. Patil, N.S. Mangale, S.V. Khare, et al., Super hard cubic phases of period VI transition metal nitrides: First principles investigation, Thin Solid Films, 517 (2008) 824-827.

[47] J. Li, X. Wang, K. Liu, et al., Crystal structures, mechanical and electronic properties of tantalum monocarbide and mononitride, Journal of Superhard Materials, 33 (2011) 173-178.

[48] M. Bielawski, K.Y. Chen, Computational Evaluation of Adhesion and Mechanical Properties of Nanolayered Erosion-Resistant Coatings for Gas Turbines, J. Eng. Gas. Turb. Power, 133 (2011) 7.

[49] X.B. Zeng, P. Peng, CALCULATION OF MECHANICAL PROPERTIES OF alpha(2)-Ti-25Al-xNb ALLOYS BY FIRST-PRINCIPLES, Acta Metallurgica Sinica, 45 (2009) 1049-1056.

[50] H. Niu, X.-Q. Chen, P. Liu, et al., Extra-electron induced covalent strengthening and generalization of intrinsic ductile-to-brittle criterion, Scientific reports, 2 (2012).

[51] S.F. Pugh, Philos. Mag., 45 (1954) 823

[52] D. Pettifor, Theoretical predictions of structure and related properties of intermetallics, Mater. Sci. Tech-lond., 8 (1992) 345-349.

[53] H. Wang, B. Wang, Q.K. Li, et al., First-principles study of the cubic perovskites BiMO3 (M=Al, Ga, In, and Sc), Phys. Rev. B, 75 (2007) 245209.

[54] H. Wang, H.T. Huang, W. Lu, et al., Theoretical prediction on the structural, electronic, and polarization properties of tetragonal Bi2ZnTiO6, J. Appl. Phys., 105 (2009) 053713.

[55] H. Wang, Layered compounds AFBiS2: superior birefringent crystals, Chinese. Phys. Lett., 32 (2015) 017801.

[56] D.G. Sangiovanni, L. Hultman, V. Chirita, et al., Effects of phase stability, lattice ordering, and electron density on plastic deformation in cubic TiWN pseudobinary transition-metal nitride alloys, Acta Mater., 103 (2016) 823-835.

[57] A.R.C. Westwood, SURFACE-SENSITIVE MECHANICAL PROPERTIES, Industrial \& Engineering Chemistry, 56 (1964) 14-25. 


\section{List of figure captions}

Fig. 1. Structure of TiN/MN superlattices. (a) $\mathrm{C} \# 1$ in $2 \times 2 \times 1$ supercell $(M=\mathrm{V})$ and (b) $\mathrm{C} \# 3$ configuration. $(M=\mathrm{W})$

Fig. 2. (a) Cauchy pressure and (b) G/B ratio of TiN/MN superlattices (SL) (C\#1 and C\#3) and Ti ${ }_{0.5} \mathrm{M}_{0.5} \mathrm{~N}$ solid solution (SS) ${ }^{[19]}$.

Fig. 3. (a, c) Brittleness and ductility trends of TiN/MN superlattices $(\mathrm{C \# 1})$ and corresponding $\mathrm{Ti}_{0.5} M_{0.5} \mathrm{~N}$ solid solution ${ }^{[19]}$. (b) The difference of

Cauchy pressure $\delta \mathrm{CP} / /$ and $\delta(\mathrm{G} / \mathrm{B})$ ratio between superlattice and corresponding solid solution.

Fig. 4. Structures of C\#3 TiN/MoN superlattice before (a) and after (b) optimization. The local distortions on Mo and N ions are observable.

Fig. 5. (a) Lattice constant $c$ of TiN (black dash line with square symbol), $M \mathrm{~N}$ (red dot line with circular symbol), and TiN/MN (blue line with triangle) superlattices at corresponding superlattice constant $a$; Shadow rectangle shows data with VIB $M$ metals. (b) Lattice constants of constraint-free TiN, $M \mathrm{~N}$, and TiN/MN C\#1 superlattices. The dotted line indicates the arithmetic mean of TiN and $M \mathrm{~N}$.

Fig. 6. Cauchy pressure $\mathrm{CP}\left(=\mathrm{C}_{12}-\mathrm{C}_{44}\right)$ of $\mathrm{TiN}$ (black dash line with square symbol), $M \mathrm{~N}$ (red dot line with circular symbol), and the in-plane Cauchy pressure $\mathrm{CP}_{\|}\left(=\mathrm{C}_{12}-\mathrm{C}_{44}\right)$ of TiN/MN (blue line with triangle) superlattices at corresponding superlattice constant $a$; Shadow rectangle shows data with VIB $M$ metals. 


\section{List of table captions}

Table 1. Lattice parameters ( $a$ in $\AA$ ), bulk modulus (B, GPa), shear modulus (G, GPa), elastic constant ( $\left.\mathrm{C}_{\mathrm{ij}}, \mathrm{GPa}\right)$, Cauchy pressure (GPa) of TiN, and $M \mathrm{~N}$ mononitrides.

Table 2. Lattice parameters ( $a, c$ in $\AA$ ), bulk modulus (B, GPa), shear modulus (G, GPa), the ratio of G/B, elastic constant $\left(\mathrm{C}_{\mathrm{ij}}\right.$, GPa), in-plane Cauchy pressure $\left(\mathrm{CP}_{\|}=\mathrm{C}_{12}-\mathrm{C}_{44}, \mathrm{GPa}\right)$, out-plane Cauchy pressure $\left(\mathrm{CP}_{\perp}=\mathrm{C}_{13}-\mathrm{C}_{66}, \mathrm{GPa}\right)$, and average Cauchy pressure $\mathrm{CPave}\left(=\left(2 \mathrm{C}_{12}+\mathrm{C}_{13}\right) / 3-\right.$ $\left.\left(2 \mathrm{C}_{44}+\mathrm{C}_{66}\right) / 3=\left(2 \mathrm{CP}_{/ /}+\mathrm{CP}_{\perp}\right) / 3, \mathrm{GPa}\right)$ of $\mathrm{C} \# 1 \mathrm{TiN} / M \mathrm{~N}$ superlattices.

Table 3. Lattice parameters $(a, c$ in $\AA$ ), macro-distortion along $c$ direction $(\delta c=c / a / \sqrt{ } 2)$, and bond-length $(\AA)$ in C\#1 TiN/MN superlattices. 500 eV. $21 \times 21 \times 15 k$-point meshes.

Table 4. Lattice parameters ( $a, c$ in $\AA$ ), macro-distortion along $c$ direction $\left(\delta c=c / a^{*} \sqrt{2}\right)$, and bond-length $(\AA)$ in $\mathrm{C \# 3}$ TiN/MN superlattices. $500 \mathrm{eV}, 12 \times 12 \times 18$ k-point meshes.

Table 5. Structural distortion of $\mathrm{TiN} / M \mathrm{~N}$ superlattices and $\mathrm{Ti}_{0.5} \mathrm{M}_{0.5} \mathrm{~N}$ solid solution. $\times$ indicates the absence of distortion and $\sqrt{ }$ indicates the presence of distortion. $\mathrm{CP}_{/ /}$stands for in-plane Cauchy pressure (GPa). The values given in brackets are $\mathrm{CP}_{/ /} / \mathrm{CP}_{\mathrm{Ref}} * 100 \%, \mathrm{CP}_{\mathrm{Ref}}$ is the value take from Ref.[19]. $500 \mathrm{eV}, 12 \times 12 \times 18 \mathrm{k}$-point meshes. 
Table I. Lattice parameters ( $a$ in $\AA$ ), bulk modulus (B, GPa), shear modulus (G, GPa), elastic constant ( $\mathrm{C}_{\mathrm{ij}}$, GPa), Cauchy pressure ( GPa) of TiN, and $M \mathrm{~N}$ mononitrides.

\begin{tabular}{|c|c|c|c|c|c|c|c|c|}
\hline & $a$ & B & G & $\mathrm{G} / \mathrm{B}$ & $\mathrm{C}_{11}$ & $\mathrm{C}_{12}$ & $\mathrm{C}_{44}$ & $\mathrm{C}_{12}-\mathrm{C}_{44}$ \\
\hline \multirow{3}{*}{ TiN } & $4.252^{*}$ & $281^{*}$ & $190^{*}$ & $0.675^{*}$ & $584^{*}$ & $128^{*}$ & $167^{*}$ & $-39^{*}$ \\
\hline & $4.254^{\mathrm{a}}$ & $290^{\mathrm{a}}$ & $200^{\mathrm{a}}$ & 0.690 & $640^{\mathrm{a}}$ & $115^{\mathrm{a}}$ & $159^{\mathrm{a}}$ & $-44^{\mathrm{a}}$ \\
\hline & 4.258 & 277 & 186 & & 596 & 119 & 153 & $-34 \mathrm{DMol}^{3}$ \\
\hline \multirow{4}{*}{$\mathrm{VN}$} & $4.124^{*}$ & $321^{*}$ & $165^{*}$ & $0.515^{*}$ & $621^{*}$ & $172^{*}$ & $126^{*}$ & $46^{*}$ \\
\hline & $4.130^{c}$ & $303^{\mathrm{c}}$ & $164^{\mathrm{c}}$ & 0.541 & $591^{\mathrm{c}}$ & $159^{\mathrm{c}}$ & $137^{\mathrm{c}}$ & $22^{c}$ \\
\hline & $4.122^{\mathrm{d}}$ & $311^{\mathrm{d}}$ & $157^{\mathrm{d}}$ & 0.505 & $616^{\mathrm{d}}$ & $163^{d}$ & $122^{\mathrm{d}}$ & $41^{\mathrm{d}}$ \\
\hline & $4.127^{\mathrm{e}}$ & & & & $621^{\mathrm{e}}$ & $167^{\mathrm{e}}$ & $117^{\mathrm{e}}$ & $50^{\mathrm{e}}$ \\
\hline \multirow{4}{*}{$\mathrm{NbN}$} & $4.453^{*}$ & $306^{*}$ & $137^{*}$ & $0.447^{*}$ & $625^{*}$ & $147^{*}$ & $69^{*}$ & $78^{*}$ \\
\hline & $4.450^{\mathrm{c}}$ & $299^{c}$ & $133^{\mathrm{c}}$ & $0.445^{c}$ & $630^{c}$ & $134^{\mathrm{c}}$ & $85^{\mathrm{c}}$ & $49^{c}$ \\
\hline & $4.422^{\mathrm{d}}$ & $304^{\mathrm{d}}$ & $130^{\mathrm{d}}$ & $0.428^{\mathrm{d}}$ & $644^{\mathrm{d}}$ & $139^{d}$ & $81^{\mathrm{d}}$ & $58^{d}$ \\
\hline & $4.427^{\mathrm{e}}$ & & & & $649^{\mathrm{e}}$ & $136^{\mathrm{e}}$ & $80^{\mathrm{e}}$ & $56^{\mathrm{e}}$ \\
\hline \multirow{6}{*}{$\mathrm{TaN}$} & $4.420^{*}$ & $332^{*}$ & $152^{*}$ & $0.459^{*}$ & $719^{*}$ & $139^{*}$ & $60^{*}$ & $78^{*}$ \\
\hline & $4.413^{\mathrm{d}}$ & $328^{\mathrm{d}}$ & $119^{\mathrm{d}}$ & 0.445 & $699^{\mathrm{d}}$ & $149^{\mathrm{d}}$ & $62^{\mathrm{d}}$ & $87^{\mathrm{d}}$ \\
\hline & $4.426^{\mathrm{e}}$ & $325^{\mathrm{e}}$ & $110^{\mathrm{e}}$ & 0.338 & $715^{\mathrm{e}}$ & $138^{\mathrm{e}}$ & $60^{\mathrm{e}}$ & $78^{\mathrm{e}}$ \\
\hline & $4.326^{\mathrm{f}}$ & $380^{\mathrm{f}}$ & & & $827^{\mathrm{f}}$ & $156^{\mathrm{f}}$ & $73^{f}$ & $83^{f}$ \\
\hline & $4.455^{\mathrm{g}}$ & $306^{\mathrm{g}}$ & $96^{\mathrm{g}}$ & $0.314^{\mathrm{g}}$ & $678^{\mathrm{g}}$ & $119^{\mathrm{g}}$ & $46^{\mathrm{g}}$ & $73^{g}$ \\
\hline & $4.341^{\mathrm{h}}$ & $402^{\mathrm{h}}$ & $189^{\mathrm{h}}$ & 0.470 & $887^{\mathrm{h}}$ & $127^{\mathrm{h}}$ & $62^{\mathrm{h}}$ & $65^{\mathrm{h}}$ \\
\hline $\mathrm{MoN}$ & $4.333^{*}$ & $331^{*}$ & $22^{*}$ & - & $568^{*}$ & $212^{*}$ & $-82^{*}$ & Unstable $^{*}$ \\
\hline \multirow{2}{*}{ WN } & $4.357^{*}$ & $356^{*}$ & $-15^{*}$ & - & $573^{*}$ & $249^{*}$ & $-129^{*}$ & Unstable $^{*}$ \\
\hline & $4.328^{\mathrm{h}}$ & $424^{\mathrm{h}}$ & & & Unstable $^{\mathrm{h}}$ & & & \\
\hline
\end{tabular}

${ }^{*}$ preset work, VASP-GGA, $500 \mathrm{eV} .30 \times 30 \times 30 k$-point meshes.

a VASP-GGA-PW91. 500 eV, 16×16×16 k-point meshes. Reference PRB 81104107 (2010). ${ }^{[18] ~ b}$ VASP-GGA-PW91. Reference Acta Mater. 59 (2011) 2121. ${ }^{[19]}$

${ }^{\mathrm{c}} \mathrm{DMol}^{3}$, GGA 12x12x12 $k$-point meshes. Reference PRB 85184106 (2012). ${ }^{[43]}$

${ }^{\mathrm{d}}$ PWSCF, GGA-PBE, $12 \times 12 \times 12$ k-point meshes. Reference J Mater Sci 48 (2013) 7642. ${ }^{[44]}$

${ }^{\mathrm{e}}$ VASP-GGA. Encut and $k$-point see Table II in Reference PRB 85 (2012) 064101. ${ }^{[45] \mathrm{f}}$ VASP-LDA. Reference Thin Solid Films 517 (2008) $824 .{ }^{[46]}$

${ }^{g}$ CASTEP-GGA. Reference J. Superhard Mater. 33 (2011)173. ${ }^{[4]}$ h FP-LMTO-LDA. Reference Materials Chemistry and Physics 143 (2013) $93 .{ }^{[42]}$ 
Table II. Lattice parameters ( $a, c$ in $\AA$ ), bulk modulus (B, GPa), shear modulus (G, GPa), the ratio of G/B, elastic constant $\left(\mathrm{C}_{\mathrm{ij}}\right.$, GPa), in-plane Cauchy pressure $\left(\mathrm{CP}_{\|}=\mathrm{C}_{12}-\mathrm{C}_{44}, \mathrm{GPa}\right)$, out-plane Cauchy pressure $\left(\mathrm{CP}_{\perp}=\mathrm{C}_{13}-\mathrm{C}_{66}, \mathrm{GPa}\right)$, and average Cauchy pressure $\mathrm{CPave}\left(=\left(2 \mathrm{C}_{12}+\mathrm{C}_{13}\right) / 3\right.$ $\left.\left(2 \mathrm{C}_{44}+\mathrm{C}_{66}\right) / 3=\left(2 \mathrm{CP}_{/ /}+\mathrm{CP}_{\perp}\right) / 3, \mathrm{GPa}\right)$ of $\mathrm{C} \# 1 \mathrm{TiN} / M \mathrm{~N}$ superlattices.

\begin{tabular}{|c|c|c|c|c|c|c|c|c|c|c|c|c|c|c|}
\hline & $a$ & $c$ & B & G & $\mathrm{G} / \mathrm{B}$ & $\mathrm{C}_{11}$ & $\mathrm{C}_{12}$ & $\mathrm{C}_{13}$ & $\mathrm{C}_{33}$ & $\mathrm{C}_{44}$ & $\mathrm{C}_{66}$ & $\mathrm{CP}_{\|}$ & $\mathrm{CP}_{\perp}$ & CPave \\
\hline TiN/VN & 2.961 & $4.194^{*}$ & 299 & 181 & 0.606 & 516 & 231 & \multirow[t]{2}{*}{148} & \multirow[t]{2}{*}{602} & 154 & \multirow[t]{2}{*}{227} & $77^{*}$ & -79 & 25 \\
\hline $\mathrm{Ti}_{0.5} \mathrm{~V}_{0.5} \mathrm{~N}$ & 4.188 & 4.188 & 312 & 170 & 0.545 & 592 & 172 & & & 144 & & $28^{\mathrm{a}}$ & $28^{\mathrm{a}}$ & $28^{\mathrm{a}}$ \\
\hline TiN/NbN & 3.089 & $4.356^{*}$ & 294 & 169 & 0.575 & 487 & 255 & \multirow[t]{2}{*}{134} & \multirow[t]{2}{*}{625} & 123 & \multirow[t]{2}{*}{242} & $132^{*}$ & -108 & 52 \\
\hline $\mathrm{Ti}_{0.5} \mathrm{Nb}_{0.5} \mathrm{~N}$ & 4.363 & 4.363 & 295 & 159 & 0.539 & 581 & 148 & & & 120 & & $28^{\mathrm{a}}$ & $28^{\mathrm{a}}$ & $28^{\mathrm{a}}$ \\
\hline TiN/TaN & 3.077 & $4.331^{*}$ & 308 & 179 & 0.581 & 510 & 276 & \multirow[t]{2}{*}{131} & \multirow[t]{2}{*}{680} & 123 & \multirow[t]{2}{*}{263} & $153^{*}$ & -132 & 58 \\
\hline $\mathrm{Ti}_{0.5} \mathrm{Ta}_{0.5} \mathrm{~N}$ & 4.348 & 4.348 & 304 & 141 & 0.464 & 559 & 176 & & & 107 & & $69^{\mathrm{a}}$ & $69^{\mathrm{a}}$ & $69^{a}$ \\
\hline TiN/MoN & 3.031 & $4.303^{*}$ & 308 & 131 & 0.424 & 431 & 346 & \multirow[t]{2}{*}{161} & \multirow[t]{2}{*}{575} & 96 & \multirow[t]{2}{*}{205} & $250^{*}$ & -44 & 152 \\
\hline $\mathrm{Ti}_{0.5} \mathrm{Mo}_{0.5} \mathrm{~N}$ & 4.301 & $4.301^{\mathrm{a}}$ & 321 & 147 & 0.458 & 655 & 153 & & & 77 & & $76^{\mathrm{b}}$ & $76^{\mathrm{b}}$ & $76^{\mathrm{b}}$ \\
\hline TiN/WN & 3.044 & $4.292^{*}$ & 324 & 128 & 0.395 & 427 & 385 & \multirow[t]{2}{*}{164} & \multirow[t]{2}{*}{637} & 89 & \multirow[t]{2}{*}{203} & $296^{*}$ & -39 & 184 \\
\hline $\mathrm{Ti}_{0.5} \mathrm{~W}_{0.5} \mathrm{~N}$ & 4.298 & $4.298^{\mathrm{a}}$ & 336 & 151 & 0.449 & 720 & 145 & & & 60 & & $85^{\mathrm{b}}$ & $85^{\mathrm{b}}$ & $85^{\mathrm{b}}$ \\
\hline
\end{tabular}

" preset work, VASP-GGA, 500 eV. $21 \times 21 \times 15 k$-point meshes.

${ }^{a}$ VASP-GGA-PW91. Reference Acta Mater. 59 (2011) 2121. ${ }^{[19] \text { b }}$ VASP-GGA-PW91. Reference PRB 81 104107 (2010). ${ }^{[18]}$

Table III. Lattice parameters ( $a, c$ in $\AA$ ), macro-distortion along $c$ direction $(\delta c=c / a / \sqrt{ } 2)$, and bond-length $(\AA)$ in $\mathrm{C} \# 1$ TiN/MN superlattices. 500 eV. $21 \times 21 \times 15 k$-point meshes.

\begin{tabular}{cccccccc}
\hline & $a$ & $c$ & $\delta c$ & Ti- $M$ & Ti-N/M-N(xy) & Ti-N/M-N(z) \\
\hline TiN/VN & 2.961 & 4.194 & 1.002 & 2.963 & 2.094 & 2.097 \\
\hline TiN/NbN & 3.089 & 4.356 & 0.997 & 3.084 & 3.184 & 2.178 \\
\hline TiN/TaN & 3.077 & 4.331 & 0.996 & 3.069 & 2.176 & 2.143 & 2.151 \\
\hline TiN/MoN & 3.031 & 4.303 & 1.004 & 3.037 & 3.039 & 2.153 & 2.146 \\
\hline TiN/WN & 3.044 & 4.292 & 0.997 & & & & \\
\hline
\end{tabular}

Table IV. Lattice parameters ( $a, c$ in $\AA$ ), macro-distortion along $c$ direction $\left(\delta c=c / a^{*} \sqrt{ } 2\right)$, and bond-length $(\AA)$ in C\#3 TiN/MN superlattices. $500 \mathrm{eV}, 12 \times 12 \times 18 \mathrm{k}$-point meshes.

\begin{tabular}{cccccccccc}
\hline & $a$ & $c$ & $\delta c$ & Ti-Ti & $M-M$ & Ti-N(xy) & Ti-N(z) & $M-\mathrm{N}(\mathrm{xy})$ & $M-\mathrm{N}(\mathrm{z})$ \\
\hline TiN/VN & 5.923 & 4.196 & 1.0018 & 2.96 & 3.18 & $2.08 / 2.13$ & 2.10 & $2.03 / 2.14$ & 2.10 \\
\hline TiN/NbN & 6.160 & 4.363 & 1.0017 & 3.05 & 3.08 & $2.10 / 2.18$ & 2.15 & $2.18 / 2.26$ & 2.20 \\
\hline TiN/TaN & 6.144 & 4.342 & 0.9993 & 3.02 & 3.07 & $2.11 / 2.19$ & 2.17 & $2.16 / 2.23$ & 2.17 \\
\hline TiN/MoN & 6.094 & 4.262 & 0.9890 & 3.06 & 3.06 & $2.12 / 2.17$ & 2.14 & $2.11 / 2.25$ & 2.15 \\
\hline TiN/WN & 6.129 & 4.250 & 0.9806 & 3.08 & 3.08 & $2.12 / 2.24$ & 2.14 & $2.12 / 2.24$ & 2.15 \\
\hline
\end{tabular}

Table V. Structural distortion of TiN/MN superlattices and $\mathrm{Ti}_{0.5} \mathrm{M}_{0.5} \mathrm{~N}$ solid solution. $\times$ indicates the absence of distortion and $\sqrt{ }$ indicates the presence of distortion. $\mathrm{CP}_{/ /}$stands for in-plane Cauchy pressure $(\mathrm{GPa})$. The values given in brackets are $\mathrm{CP}_{/ /} / \mathrm{CP}_{\mathrm{Ref}} * 100 \%, \mathrm{CP}_{\mathrm{Ref}}$ is the value take from Ref.[19]. $500 \mathrm{eV}, 12 \times 12 \times 18$ k-point meshes.

\begin{tabular}{|c|c|c|c|c|c|c|c|}
\hline \multirow{2}{*}{ Model } & \multirow{2}{*}{ Macro-distortion } & \multirow{2}{*}{ Micro-distortion } & \multicolumn{5}{|c|}{$\mathrm{CP}_{/ /}$} \\
\hline & & & $\mathrm{V}$ & $\mathrm{Nb}$ & $\mathrm{Ta}$ & Mo & $W$ \\
\hline C\#3 (Ref.[19]) & $x$ & $x$ & 28 & 28 & 69 & 76 & 85 \\
\hline C\#1 (Present) & $\sqrt{ }$ & $x$ & $77(275 \%)$ & $132(471 \%)$ & $153(222 \%)$ & $250(329 \%)$ & $296(348 \%)$ \\
\hline C\#3 (Present) & $\sqrt{ }$ & $\sqrt{ }$ & $87(311 \%)$ & $129(461 \%)$ & $136(197 \%)$ & $201(264 \%)$ & $194(228 \%)$ \\
\hline
\end{tabular}



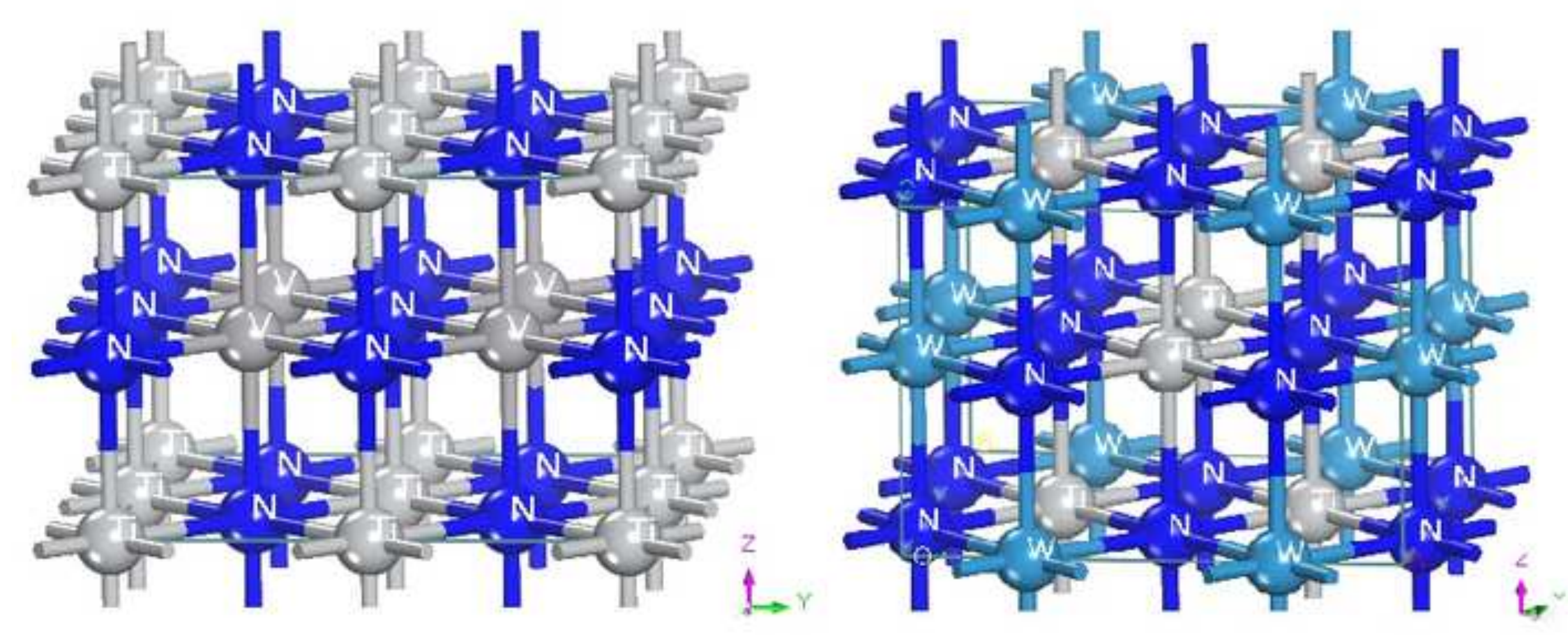
(b) 0.20

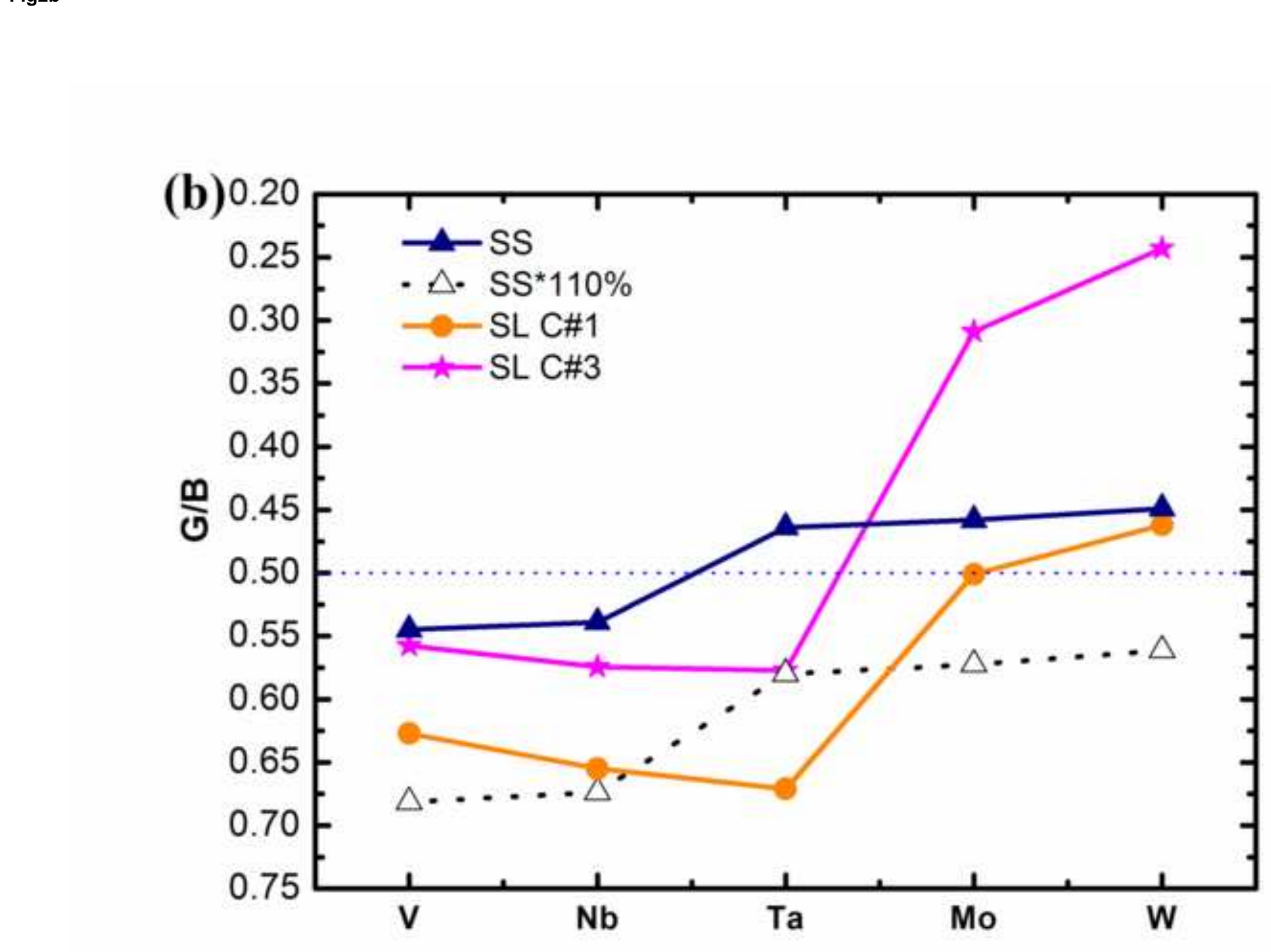




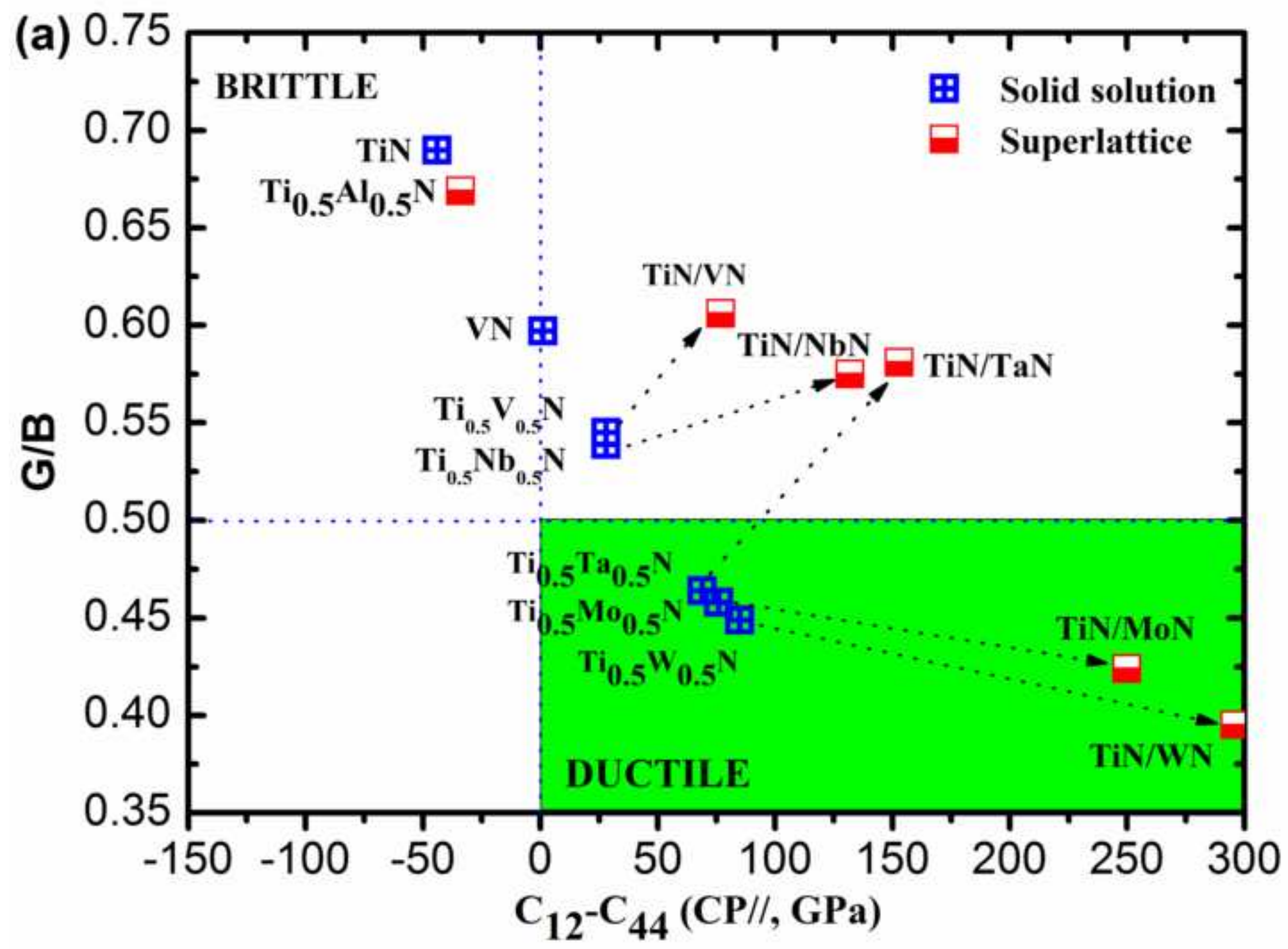



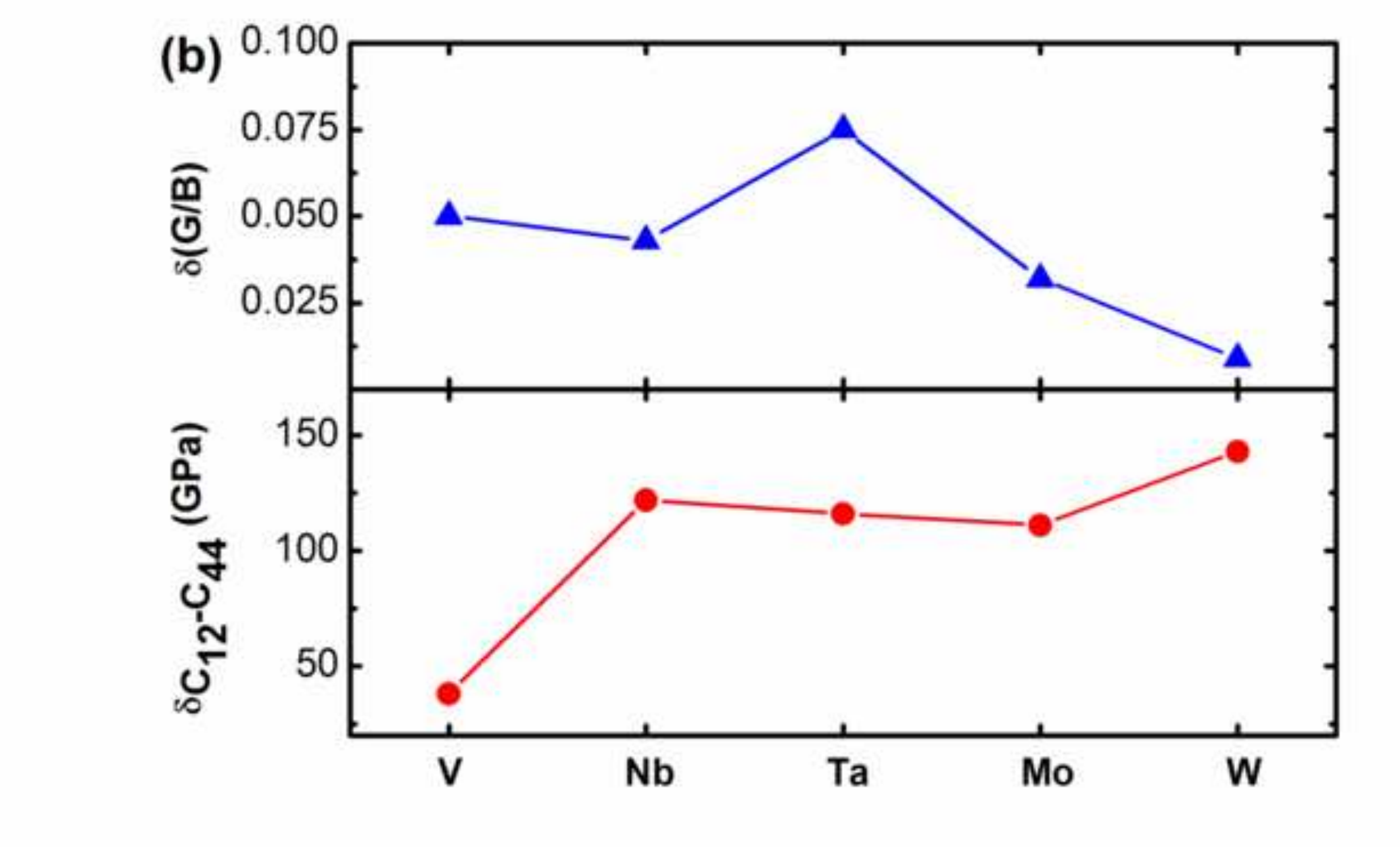

(1)
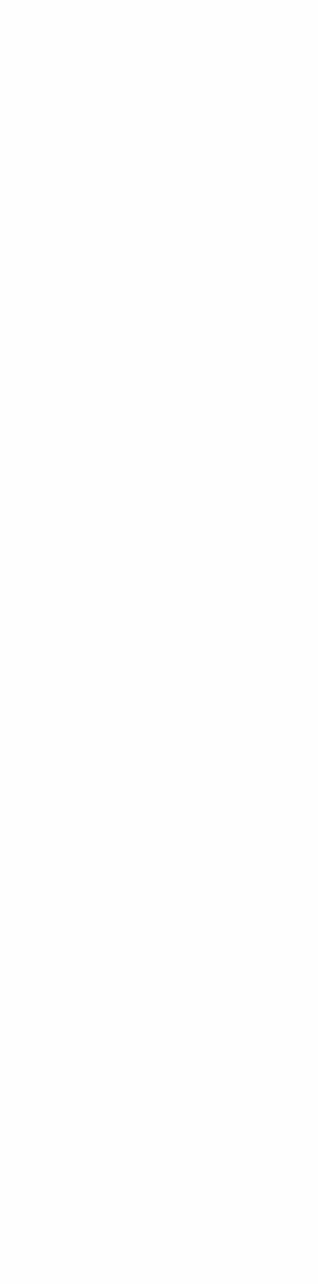

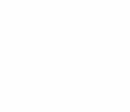
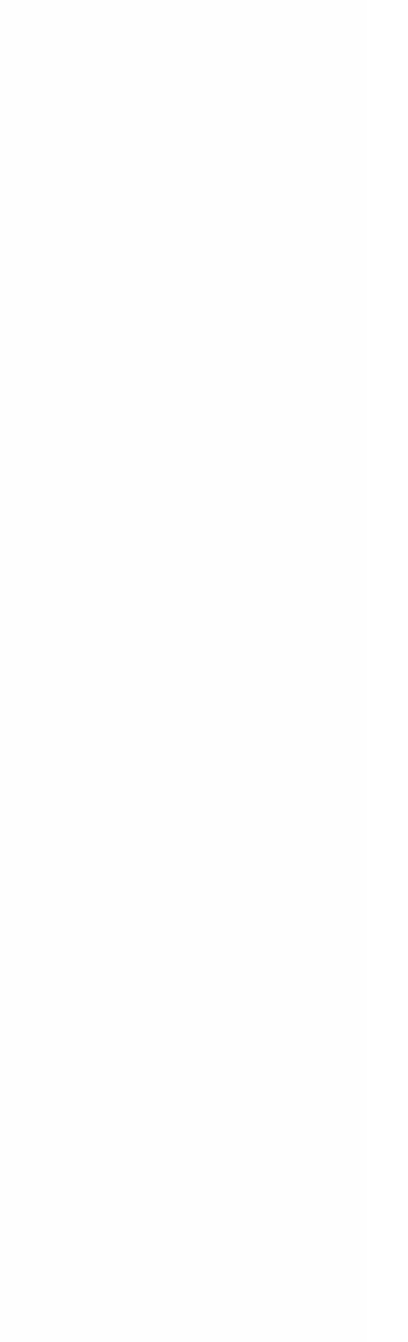


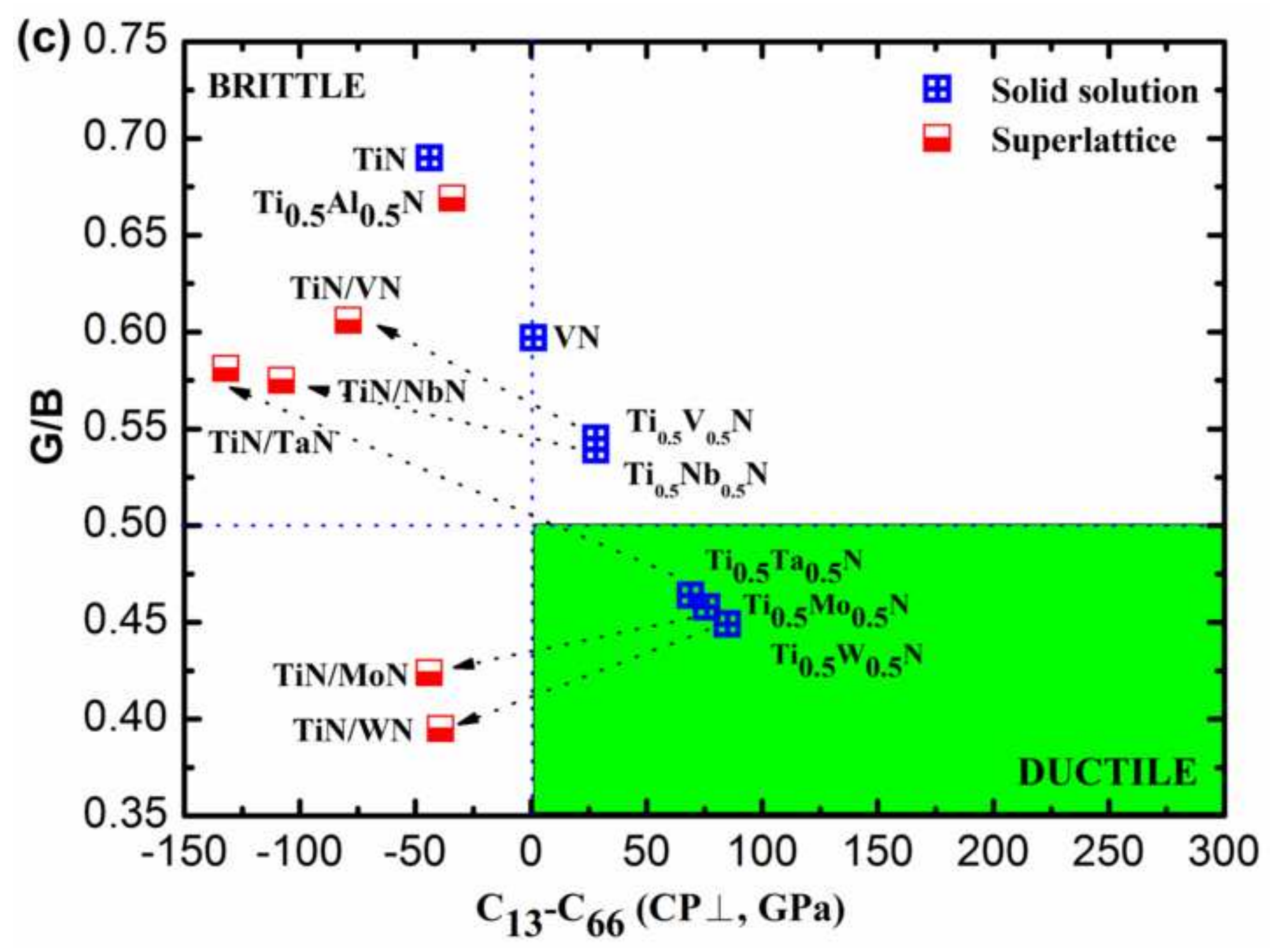




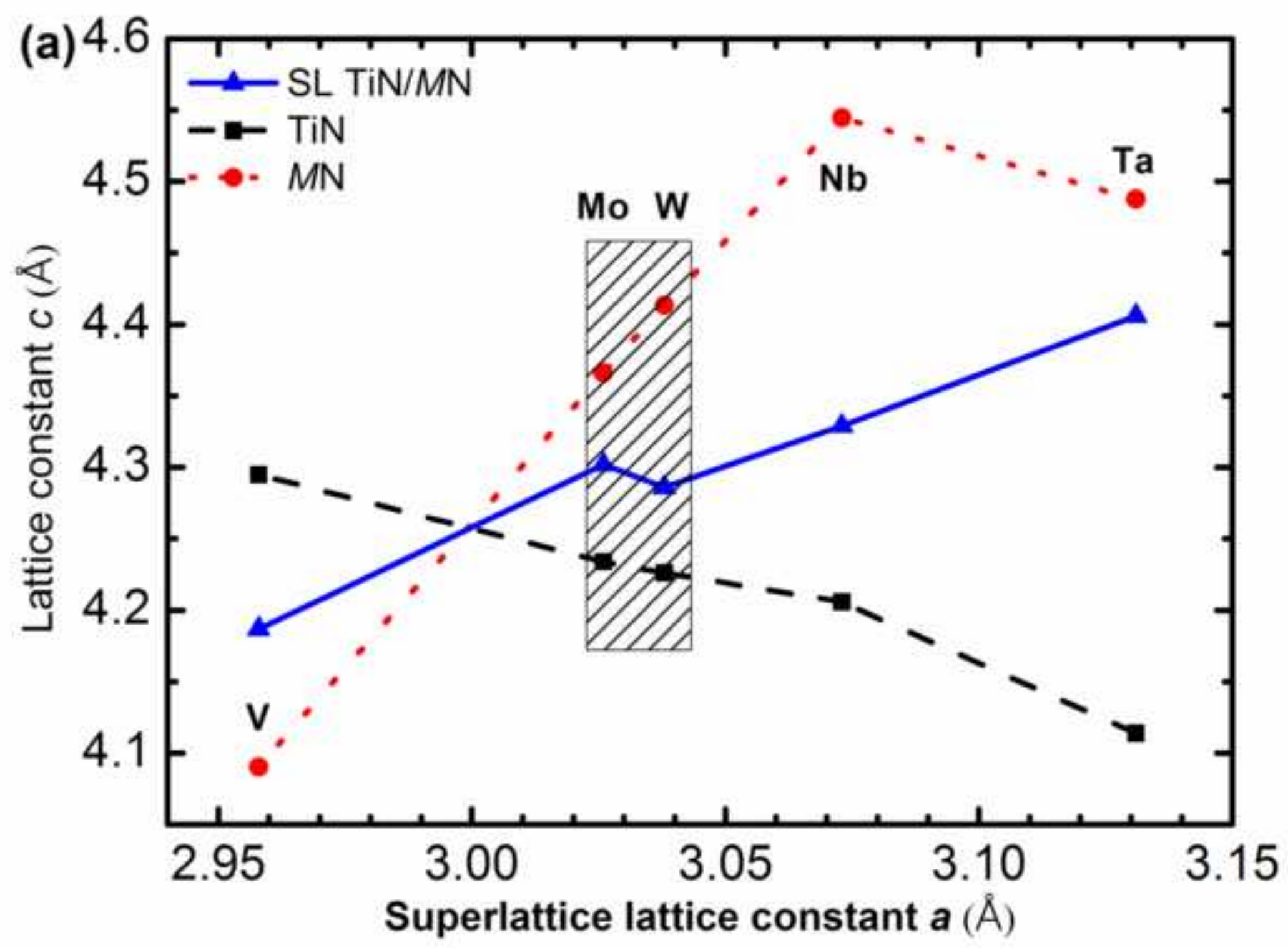




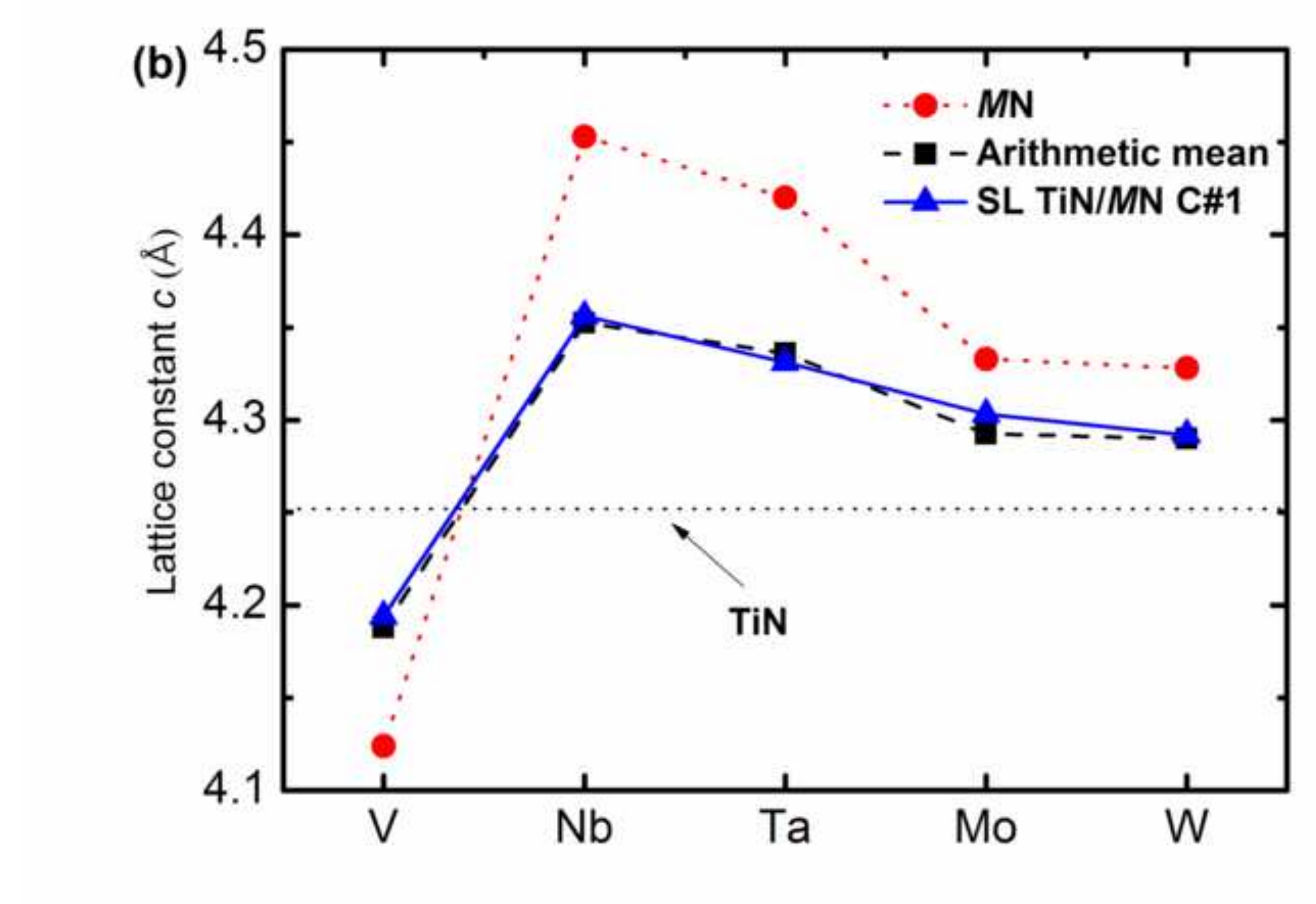

.

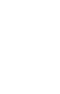

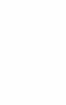




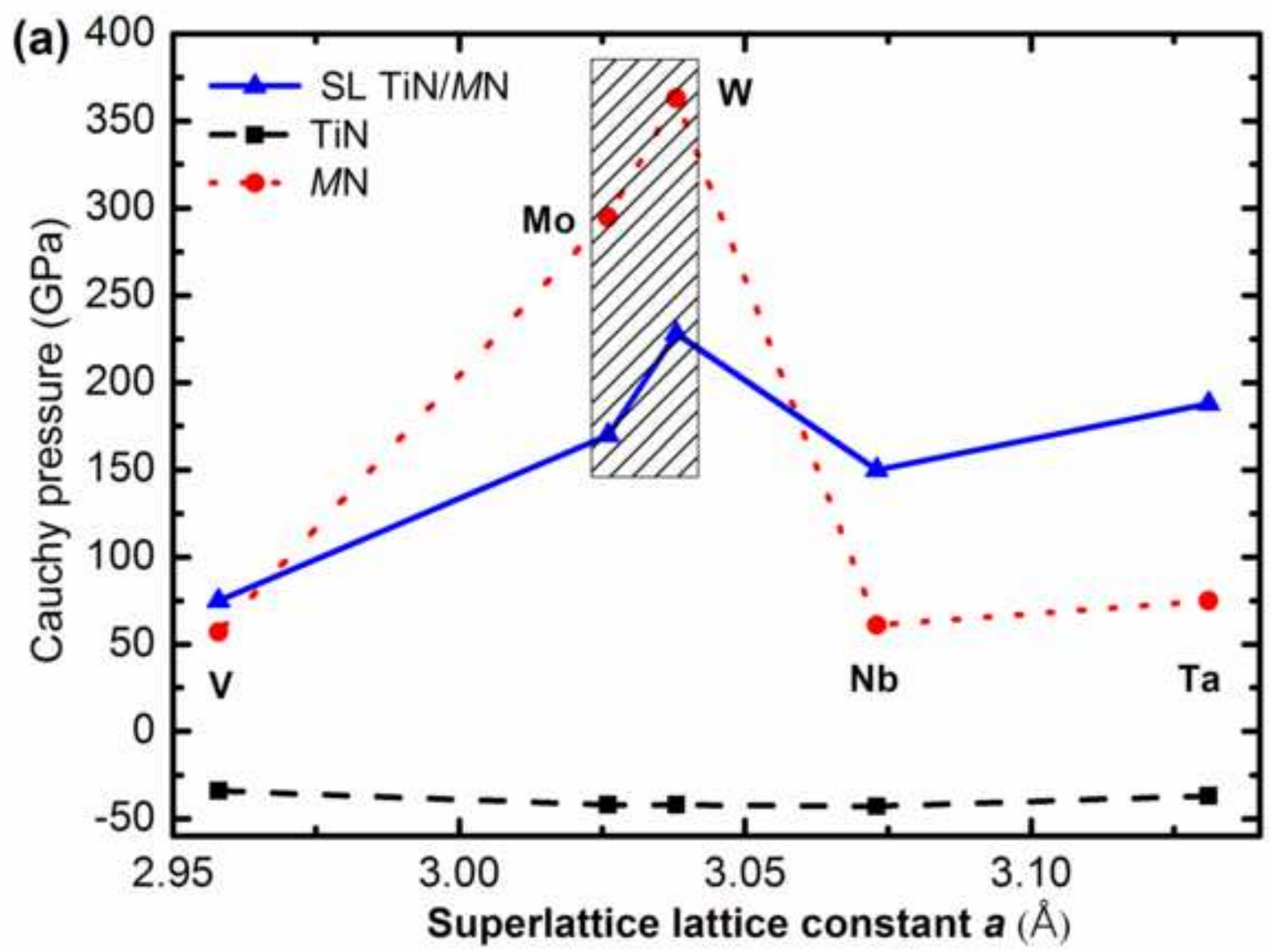




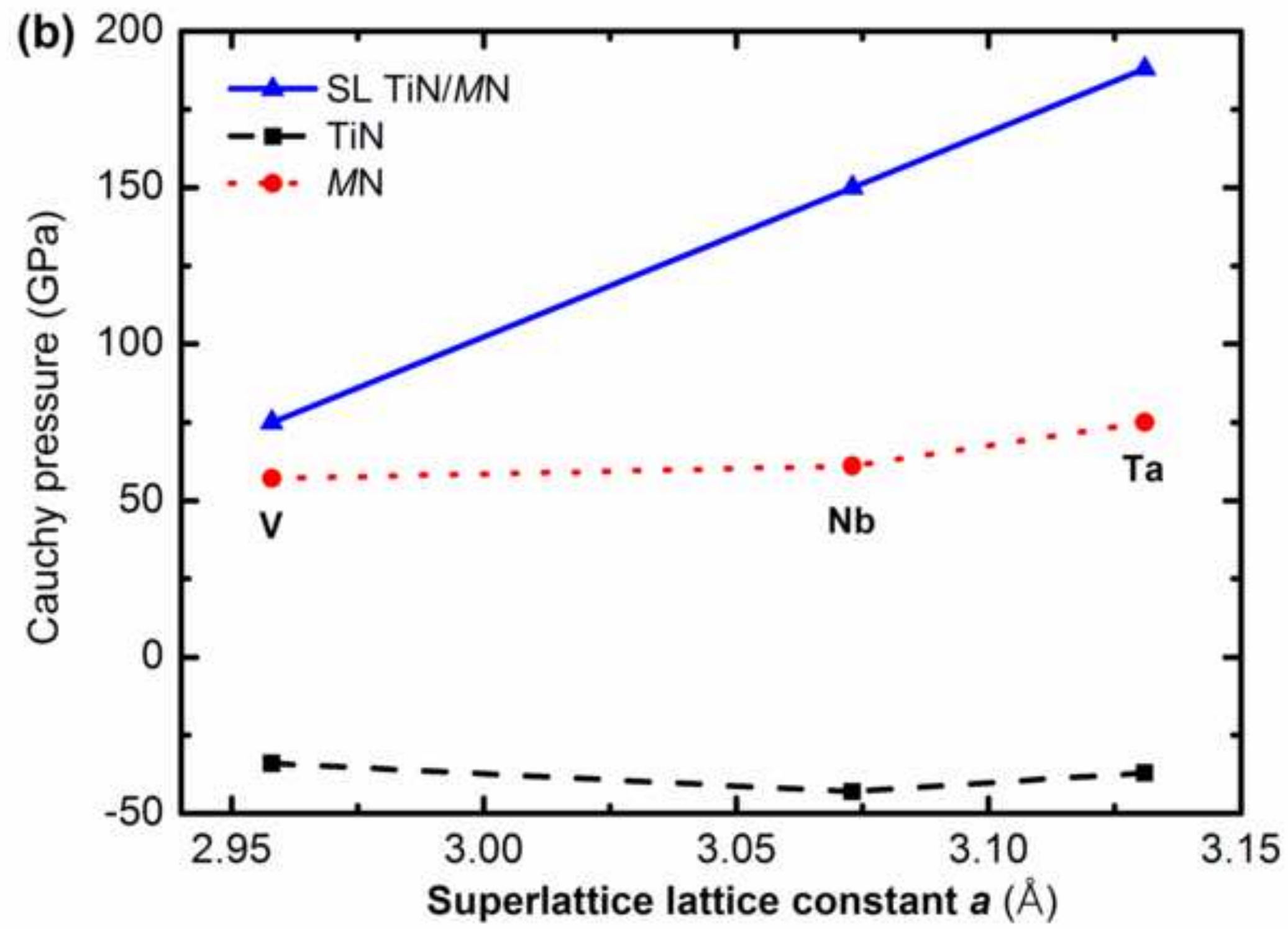

